\title{
Evaluación y análisis de la calidad de un servicio de apoyo desde la perspectiva del usuario: Primer paso hacia la confiabilidad
}

\author{
Recepción: Febrero de 2007 / Aceptación: Mayo de 2007
}

(1) Jorge Pérez Rave
(2) Carlos Parra Mesa

\begin{abstract}
RESUMEN
Este artículo exhibe la evaluación y el análisis de la calidad del servicio de soporte técnico de la Facultad de Ingeniería de la Universidad de Antioquia (Colombia). La metodología integra elementos de: investigación cualitativa, estadística multivariable, calidad, teoría de probabilidad y confiabilidad de sistemas. En el presente artículo se discute que, el análisis de la calidad debe verse como un primer paso hacia el análisis de la confiabilidad, el cual constituye una práctica aún mas efectiva para las organizaciones, por permitir conocer el comportamiento que caracteriza a la calidad a lo largo del tiempo, las fallas del servicio, la severidad de las mismas, e identificar con mayor certeza hacia donde deben orientarse los esfuerzos de mejoramiento.
\end{abstract}

Palabras clave: calidad del servicio, confiabilidad de servicios, análisis multivariado, dimensiones de calidad, satisfacción del cliente, servicio de soporte técnico.

ASSESSMENT AND ANALYSIS OF THE QuALITY OF A SUPPORT SERVICE DONE FROM USERS' STANDPOINT: FIRST STEP FOR RELIABILITY

ABSTRACT

The assessment and analysis of the technical support service's quality of the Engineering Department of the University of Antioquia (Colombia) is show in this article. The aforementioned methodology integrates elements of: qualitative research, multivariable statistics, quality, probability theory and system reliability. In the present article is discussed that, quality analysis should be seen as the first step for reliability analysis, which is more effective for firms because it allows them to know the behavior of quality throughout time, the flaws in their service, their severity, and the best direction in which the improvement activities must be oriented.

Key words: service quality, service reliability, multivariate analysis, quality dimensions, customer satisfaction, technical support service.

\section{INTRODUCCIÓN}

El creciente desarrollo tecnológico y de las comunicaciones, exige de las universidades una serie de recursos computacionales destinados a apoyar su efectivo funcionamiento y la formación de profesionales capaces de satisfacer las necesidades de las organizaciones empresariales y de la sociedad en general.

La Universidad de Antioquia (Colombia) no es ajena a esta realidad, y por ello cuenta a nivel de sus facultades con personal encargado de velar por el buen funcionamiento de los mencionados recursos. La Facultad de Ingeniería, en particular, posee el Departamento de Recursos de Apoyo e Informática (DRAI), un departamento de carácter administrativo creado en 1992 que dentro de su portafolio de servicios ofrece el soporte técnico, un servicio cuya razón de ser es velar para que cada usuario de la Facultad, que tenga a su cargo un equipo de cómputo de la Universidad, pueda utilizarlo eficaz y eficientemente cada vez que lo requiera.

Estos equipos están destinados a labores docentes, administrativas, de investigación y de extensión, por lo cual, una falla en los mismos que no sea atendida oportunamente y con la suficiente efectividad, puede ocasionar desde simples minutos de espera por parte del usuario, hasta la pérdida de información relevante producto de largos periodos de investigación.

Este propósito hace imperante identificar las necesidades de los usuarios del servicio de soporte técnico del DRAI, y al mismo tiempo, evaluar el grado en que se satisfacen, con la finalidad de posibilitar el establecimiento de directrices de mejora que contribuyan al aumento de la satisfacción de los usuarios y al efectivo desarrollo de las actividades en la Facultad de Ingeniería.

El presente artículo ofrece la evaluación y el análisis de la calidad del servicio de soporte técnico del DRAI, desde la perspectiva del usuario, y constituye la primera parte de los resultados arrojados por una investigación realizada por los autores en la Facultad de Ingeniería de la Universidad de Antioquia (Colombia). En dicha investigación se desarrolló y se aplicó al mencionado servicio, una metodología que permite evaluar y analizar la confiabilidad de servicios desde la perspectiva del cliente, siendo la evaluación y el análisis de la calidad, el primer paso para avanzar hacia la confiabilidad, entendiéndose ésta última como "calidad a través del tiempo" (Escobar et al 2003).

\footnotetext{
(1) Ingeniero Industrial. Miembro de los grupos de investigación: Gestión de la Calidad, Ingeniería de Formación y, Ciencia y Tecnología Biomédica (CTB). Departamento de Ingeniería Industrial. Universidad de Antioquia, Colombia.

Tecnologia Biomédica (CTB),

(2) Estadístico MSc. Director del Grupo Ciencia y Tecnoloía Biomédica (CTB), Profesor del Departamento de Ingeniería Industrial.

Universidad de Antioquia, Colombia
}

E-mail:cmparra@udea.edu.co 


\section{MARCO DE REFERENCIA}

En la última década la atención al cliente se ha tornado vital para la supervivencia de toda empresa, razón por la cual es necesario generar incesantes esfuerzos para garantizar la satisfacción de las necesidades de sus clientes; de ahí que la calidad de los productos y servicios se convierte en una característica indispensable para la competitividad de una organización.

El término calidad ha sido definido de diversas maneras. De acuerdo con Juran y Gryna (1993) calidad es "conjunto de características que satisfacen las necesidades del consumidor", Montgomery (1996) se refiere a ella como el grado hasta el cual los productos satisfacen las necesidades de sus consumidores y, Andriani C. et al (2003) la define como "atender los anhelos, necesidades y aspiraciones de los clientes".

Como puede observarse en las anteriores definiciones, el concepto calidad está íntimamente asociado a la satisfacción del cliente. Chias (1999) define la satisfacción del cliente como "el resultado de la evaluación posterior al uso, que realiza el usuario sobre el valor recibido"; y de acuerdo con Coyne (Larrea, P. 1991) existen cinco estados de satisfacción, los cuales se presentan en el cuadro 1.

Consecuentes con lo anterior, evaluar la calidad del servicio de soporte técnico del DRAl, puede interpretarse como evaluar el grado en que se satisfacen las necesidades de los usuarios, pero para

Cuadro 1. Estados de satisfacción

\begin{tabular}{|c|l|}
\hline Estados & \multicolumn{1}{|c|}{ Descripción } \\
\hline Excitación & $\begin{array}{l}\text { El usuario se ha llevado una sorpresa } \\
\text { agradable porque las expectativas de } \\
\text { éxito eran bajas o el nivel de esfuerzo } \\
\text { esperado por el usuario era alto. }\end{array}$ \\
\hline Satisfacción & $\begin{array}{l}\text { Las expectativas han sido igualadas } \\
\text { pero no superadas. La transacción se } \\
\text { ha desarrollado perfectamente y el nivel } \\
\text { de esfuerzo ha sido el esperado. }\end{array}$ \\
\hline Irritación & $\begin{array}{l}\text { La transacción ha ocurrido felizmente } \\
\text { pero el comportamiento del proveedor } \\
\text { no ha sido apropiado. }\end{array}$ \\
\hline Insatisfacción & $\begin{array}{l}\text { La transacción no ha tenido éxito a } \\
\text { pesar del esfuerzo exigido. }\end{array}$ \\
\hline Enfado & $\begin{array}{l}\text { La transacción no ha tenido éxito a pesar } \\
\text { de un esfuerzo por parte del cliente, } \\
\text { notablemente superior al esperado por } \\
\text { culpa del proveedor que podía habérselo } \\
\text { evitado }\end{array}$ \\
\hline
\end{tabular}

esto se requiere identificar en ellos, cuáles son las necesidades importantes, también conocidas como dimensiones de calidad. Kennedy y Young (1989) establecen cinco dimensiones de calidad para servicios de apoyo, las cuales se presentan en el cuadro 2 .

Las anteriores dimensiones pueden ser adaptadas al servicio de soporte técnico de la Facultad de Ingeniería, teniendo en cuenta que en este caso el cliente es todo usuario de la Facultad que tenga a su cargo un equipo de cómputo de la Universidad de Antioquia.

Para identificar las dimensiones de calidad del servicio puede emplearse el método de los incidentes críticos, entendiéndose como incidente crítico, un ejemplo específico del desempeño organizacional desde la perspectiva del usuario. En este método, luego de colectar los incidentes críticos, se forman grupos y se redactan frases que reflejen el contenido de los mismos, las cuales se denominan elementos de satisfacción, y a partir de ellos, se definen las dimensiones de calidad del servicio. El procedimiento de los incidentes críticos se describe en el cuadro 3 (Hayes B.1999).

La determinación de la estructura de las dimensiones de calidad, consiste en conformar conglomerados de elementos de satisfacción estrechamente relacionados entre sí, cada uno de los cuales define una dimensión de calidad. Para tal fin existen diversas técnicas, tanto de carácter subjetivo como objetivo; entre las primeras se encuentra la técnica de acuerdo

\begin{tabular}{|c|l|}
\multicolumn{2}{|c|}{ Cuadro 2. Dimensiones de calidad para } \\
servicios de apoyo.
\end{tabular}


Cuadro 3. Método de los incidentes críticos.

\begin{tabular}{|l|}
\hline \multicolumn{1}{|c|}{ Pasos } \\
\hline 1. Capturar incidentes críticos generados por los usuarios \\
\hline 2. Clasificar los incidentes críticos por grupos \\
\hline $\begin{array}{l}\text { 3. Redactar elementos de satisfacción para cada grupo } \\
\text { de incidentes críticos }\end{array}$ \\
\hline 4. Determinar la estructura de las dimensiones de calidad \\
\hline
\end{tabular}

entre jueces (Hayes, B. 1999) y, entre las segundas, el "análisis de factores" (Dallas, E. 1998).

Es de resaltar que, para medir la calidad del servicio deben considerarse una serie de características intangibles y la manera como éstas son percibidas por el usuario. Bolton, R. N. y Drew, J.H (1991) mencionan que operacionalmente la calidad es una actitud; por tanto, puede ser valorada como la sumatoria de las percepciones sobre los atributos del servicio (Churchill, G. y Surprenant, C. 1982). De acuerdo con Oskamp (1977), "una actitud, es una predisposición aprendida para responder conscientemente de manera favorable o desfavorable ante un objeto de sus símbolos".

Para medir las actitudes se han desarrollado diversos métodos, entre los que cabe mencionar: Intervalos de Thurstone (Thurstone, L. L. 1931) y la escala de Likert (Likert, R. 1932). La escala de Thurstone es más laboriosa que la de Likert, y esta última produce coeficientes de confiabilidad más altos y con menos elementos (Chang, L. 1994). Sin embargo, a pesar de contar con buenos instrumentos para medir las actitudes de los clientes, en este caso de los usuarios, es importante aclarar que el resultado de la medición debe interpretarse como "síntomas" y no como "hechos" (Padua, 1979).

Parra et al (2001), en una investigación realizada sobre las calificaciones del desempeño docente, asignadas por los estudiantes, empleando la escala Likert resalta: "ésta debe tomarse como un registro de percepciones u opiniones y en ningún caso como una calificación exacta (es decir, con variabilidad cero y sin sesgo)".

En este sentido, si bien es cierto que empleando la escala Likert no es posible conocer el nivel exacto de satisfacción de los usuarios, si se puede afirmar que mediante ella se obtiene información muy confiable del concepto subyacente "satisfacción". Esto hace posible además, que sea factible hacer inferencia estadística sobre las relaciones con las dimensiones
Figura 1. Relación jerárquica entre la calidad global, las dimensiones y los elementos de satisfacción.

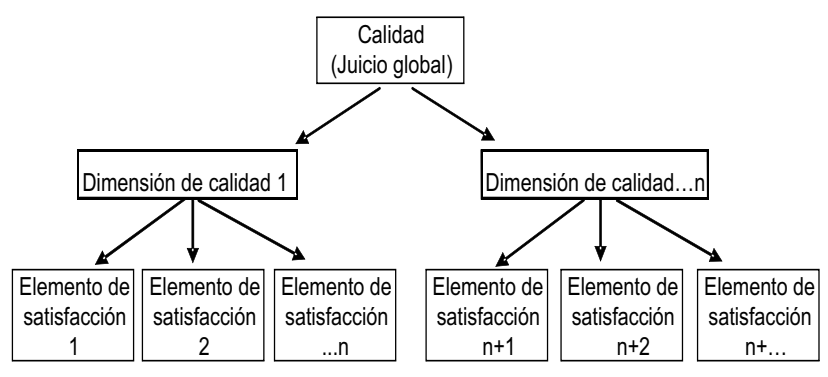

de calidad y estimar adecuadamente el nivel de calidad del servicio.

En la figura 1 se muestra la relación jerárquica entre la calidad global, las dimensiones de calidad y los elementos de satisfacción que las definen. De ella puede observarse que la satisfacción global de las necesidades de los usuarios, depende del grado en que se satisfacen cada una de las dimensiones de calidad del servicio, el cual a su vez, obedece a la satisfacción percibida por el usuario en cada uno de los elementos de satisfacción.

Es importante resaltar, que las dimensiones de calidad deben ser independientes entre sí, y aunque ello no se consigue totalmente ya que depende no sólo de la elaboración del cuestionario, sino también de factores ajenos a los encuestados, se debe buscar que la correlación entre ellas sea mínima (Pérez, C. J, 2002).

\section{METODOLOGÍA}

\section{Revisión literaria}

En primer lugar, se adelantó una revisión literaria con el fin de identificar dimensiones de calidad para servicios de apoyo, así como los elementos de satisfacción que las definen. Lo anterior, con el fin de tener una base para posteriormente denominar los elementos de satisfacción característicos del servicio de soporte técnico del DRAI y sus dimensiones de calidad.

\section{Identificación y clasificación de los incidentes críticos}

Con el ánimo de diseñar el cuestionario para medir el grado en que el servicio objeto de estudio satisface las necesidades de sus usuarios, se realizó una investigación cualitativa para colectar incidentes críticos; es decir, aseveraciones favorables y desfavorables del servicio. Para ello, siguiendo el procedimiento de los incidentes críticos (Hayes, B. 1999), se entrevistaron 19 de los usuarios, atendidos 
Cuadro 4. Items del cuestionario

\begin{tabular}{|l|}
\hline \multicolumn{1}{|c|}{ Ítems } \\
\hline Dejaron el servicio empezado y volvieron al rato. \\
\hline $\begin{array}{l}\text { El personal demostró estar capacitado para } \\
\text { solucionar el problema. }\end{array}$ \\
\hline $\begin{array}{l}\text { Me prestaron el servicio a una hora conveniente } \\
\text { para mí. }\end{array}$ \\
\hline $\begin{array}{l}\text { La página Web estaba caída cuando necesité el } \\
\text { servicio }\end{array}$ \\
\hline $\begin{array}{l}\text { La persona que me atendió, perdió tiempo en } \\
\text { actividades que no tenían que ver con el servicio }\end{array}$ \\
\hline Me solucionaron el problema \\
\hline $\begin{array}{l}\text { Me prestaron ayuda desde el principio hasta el } \\
\text { final del servicio }\end{array}$ \\
\hline $\begin{array}{l}\text { Tuve que esperar mucho tiempo para que vinieran } \\
\text { a atenderme }\end{array}$ \\
\hline $\begin{array}{l}\text { Cuando solicité el servicio me atendieron antes } \\
\text { de } 24 \text { horas }\end{array}$ \\
\hline Me describieron el problema y la solución \\
\hline Me resolvieron todas las dudas que tenía \\
\hline $\begin{array}{l}\text { Pude contactarme fácilmente con el DRAl para } \\
\text { solicitar el servicio }\end{array}$ \\
\hline $\begin{array}{l}\text { La persona que me atendió terminó el trabajo } \\
\text { rápidamente }\end{array}$ \\
\hline $\begin{array}{l}\text { La persona que me atendió prestó atención } \\
\text { cuando le hablé }\end{array}$ \\
\hline La persona que me atendió no fue amable \\
\hline $\begin{array}{l}\text { La calidad del servicio cumplió con mis } \\
\text { expectativas }\end{array}$ \\
\hline
\end{tabular}

entre el $27 / 08 / 05$ y el $06 / 09 / 05$, los cuales fueron seleccionados de modo que estuviesen representadas las actividades realizadas en la Facultad, las dependencias atendidas por el DRAI, los monitores que prestan el servicio y el género de los usuarios. Dicha investigación permitió colectar 123 incidentes críticos, los cuales fueron clasificados en grupos, cada uno de ellos representado por medio de una frase que corresponde a un elemento de satisfacción.

\section{Construcción del cuestionario}

En esta fase se diseñó el cuestionario para evaluar la calidad del servicio, el cual fue sometido a revisión de expertos externos al proyecto para verificar el cumplimiento de requisitos de redacción, entendimiento y propósito de la medición. El mencionado instrumento consta de 16 ítems favorables y desfavorables del servicio medidos en un escalamiento tipo Likert, que va desde totalmente de acuerdo, hasta totalmente en desacuerdo. Los primeros 15 ítems corresponden a los elementos de satisfacción definidos en la etapa anterior $y$, se agregó un último ítem, que hace referencia a la calidad general percibida por cada usuario ("la calidad del servicio cumplió mis expectativas"), el cual se empleará posteriormente para analizar las relaciones con las dimensiones de calidad. En el cuadro 4 se presentan dichos ítems.

\section{Aplicación del cuestionario}

Para obtener datos sobre la percepción de los usuarios frente a la satisfacción de sus necesidades, que permitan identificar con claridad la estructura de las dimensiones de calidad, y al mismo tiempo, estimar el nivel de calidad del servicio, el cuestionario elaborado se aplicó en el grupo de usuarios que durante Octubre 03 de 2005 y Febrero 01 de 2006 recibieron el soporte técnico. En vista que en tal periodo un mismo usuario podría recibir varios servicios, se realizó una sola encuesta por usuario, a fin de garantizar que las percepciones capturadas sobre la calidad durante los servicios prestados fuesen independientes entre sí. Otra razón importante para tal decisión, fue la de evitar generar malestar al usuario realizándole más de una encuesta, lo que de hacerse, aumentaría la posibilidad de que éste responda en las siguientes encuestas de una manera no razonada.

Consecuentes con lo anterior, la población a estudiar estuvo conformada por los 80 binomios usuarioservicio, atendidos por el DRAI durante el periodo de estudio. Es de resaltar que la población se abordó en su totalidad, lo cual significa que en ausencia de errores, los indicadores estadísticos corresponden a los parámetros poblacionales.

\section{Tabulación y control de calidad}

Recolectados los datos, se elaboró una típica matriz en Excel, se realizó la tabulación de los mismos, y luego de ello, se efectúo control de calidad de la tabulación para el $80 \%$ de los cuestionarios, lográndose una satisfacción plena.

\section{Identificación de las dimensiones de calidad}

Para determinar la cantidad de dimensiones de calidad que subyacen en los ítems sometidos a valoración, mediante el software SPSS 14.0 se efectuó inicialmente el análisis de componentes principales (Dallas E, 1998) y se seleccionaron aquellos factores con valor propio mayor que 1 . Luego de ello, con el fin de facilitar la interpretación de las dimensiones y determinar la estructura de cada una de ellas, se llevó a cabo el procedimiento de rotación Varimax, considerándose como cargas 
importantes, aquellas con valor absoluto superior a 0,4 (Dallas E, 1998). Con este procedimiento se busca que las cargas de un ítem original estén cercanas a 1 en una sola dimensión y próximas a cero en las demás.

\section{Consistencia interna de las dimensiones de calidad}

Con el propósito de analizar la validez y el carácter confiable de la estructura de las dimensiones de calidad y del cuestionario completo, se recurrió al coeficiente de consistencia interna Alpha Cronbach $(\alpha)$, cuya relación matemática se presenta en la ecuación 1(Carmines y Zeller, 1979).

$$
\alpha=\frac{N}{(N-1)\left[\frac{1-\sum S^{2}\left(Y_{i}\right)}{S^{2} X}\right]} \quad \text { Ec. } 1
$$

Donde "N" es la cantidad de ítems, $\sum S^{2}\left(Y_{i}\right)$ corresponde a la sumatoria de las varianzas de los ítems y " $S^{2} X$ " es igual a la varianza de toda la escala de interés. Es de resaltar que para calcular la confiabilidad de una determinada dimensión, sólo han de emplearse los ítems que la conforman.

\section{Cálculo de la satisfacción de los usuarios con las dimensiones de calidad}

El grado de satisfacción de los usuarios con cada dimensión de calidad se determinó a través del promedio aritmético ponderado entre los puntajes medios de los ítems que conforman cada dimensión, empleándose como factor de ponderación, la carga de dichos ítems en la primera componente principal de la matriz de extracción, resultante del análisis de componentes principales previamente efectuado. Esta carga puede interpretarse como la importancia de los ítems en el constructo subyacente "satisfacción". La ecuación 2 exhibe la relación matemática empleada para tal fin.

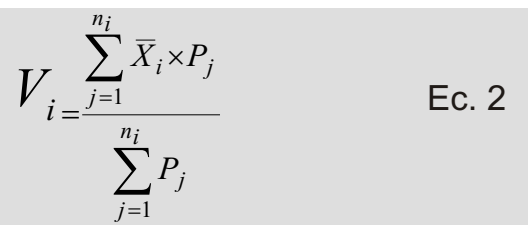

Donde " $n_{i}$ " es la cantidad de ítems que conforman la i-ésima dimensión de calidad, " $P_{j}$ " corresponde a la carga del ítem j en la primera componente principal de la matriz de extracción, " $\bar{X}_{j}$ " es la puntuación media del j-ésimo ítem y, " $V_{i}$ " es el grado de satisfacción de los usuarios con la i-ésima dimensión.
Importancia relativa de las dimensiones de calidad

El peso de cada dimensión en el constructo subyacente "calidad", se determinó mediante la correlación entre el grado de satisfacción de los usuarios con las dimensiones y el ítem del cuestionario ("la calidad del servicio cumplió con mis expectativas"), el cual hace referencia a la calidad general expresada por el usuario, considerándose de mayor importancia relativa, aquellas dimensiones más correlacionadas con dicho ítem (Hayes, B. 1999). Luego de ello, para vislumbrar de una manera más objetiva el peso de cada dimensión de calidad, se procedió a normalizar los valores de las correlaciones, mediante la siguiente ecuación.

$$
\begin{array}{ll}
K_{i}=\frac{\rho_{i}}{\sum_{1}^{5} \rho_{i}} x 100 \quad \text { Ec. } 3
\end{array}
$$

Siendo " $K_{i}$ " la importancia relativa de cada dimensión y, " $\rho_{i}$ " la correlación entre la i-ésima dimensión y el ítem alusivo a la calidad general expresada por los usuarios.

\section{Cálculo de la calidad del servicio}

La calidad del servicio se determinó mediante la siguiente ecuación funcional:

$$
C=\sum_{i=1}^{5} K_{i} \times V_{i} \quad \text { Ec. } 4
$$

Siendo " $K_{i}$ " la importancia relativa de la i-ésima dimensión de calidad y "” la calidad del servicio, con $i=1, \ldots 5$.

Es decir, siendo teóricamente independientes las dimensiones de calidad, y teniendo en cuenta que la importancia relativa de cada una de ellas puede diferir de manera significativa, la calidad global del servicio puede estimarse razonablemente mediante la suma ponderada de la satisfacción de los usuarios con las dimensiones de calidad, siendo el factor de ponderación la importancia relativa de cada una de ellas (Pérez, C. J. 2002).

\section{RESULTADOS Y ANÁLISIS}

\section{Estructura de las dimensiones de calidad}

Luego de efectuado el análisis de componentes principales y la rotación Varimax, se halló que la calidad del servicio de soporte técnico del DRAl está en función de cinco dimensiones de calidad, con una 
explicación del $71 \%$ de la varianza total. Este porcentaje de explicación se considera satisfactorio para los fines del estudio, teniendo presente la intangibilidad de los servicios, la subjetividad que caracteriza las percepciones de los usuarios y los factores incontrolables que intervienen en ella, como por ejemplo el estado de ánimo del usuario. Además, esta cantidad de dimensiones es coherente con lo planteado por la literatura para los servicios de apoyo (ver cuadro 2). En el cuadro 5 se exhibe las dimensiones de calidad del servicio, la carga de los ítems luego de efectuar la rotación Varimax y el porcentaje de varianza explicada por cada dimensión.

\section{Satisfacción de los usuarios con las dimensiones de calidad}

Inicialmente, para cada dimensión de calidad se muestra el puntaje de los ítems que la conforman, resaltando que, a fin de facilitar la interpretación,

Cuadro 5. Estructura de las dimensiones de calidad.

\begin{tabular}{|c|c|c|c|c|}
\hline $\mathbf{N}^{\mathbf{0}}$ & Dimensión & Definición & Elementos & Carga \\
\hline \multirow{6}{*}{1} & \multirow{6}{*}{ Capacidad de apoyo } & \multirow{6}{*}{$\begin{array}{l}\text { Grado hasta el cual } \\
\text { el proveedor } \\
\text { demuestra } \\
\text { capacidad para } \\
\text { terminar el trabajo } \\
\text { total }\end{array}$} & $\begin{array}{l}\text { El personal demostró estar capacitado } \\
\text { para solucionar el problema }\end{array}$ & 0.81 \\
\hline & & & $\begin{array}{l}\text { Me resolvieron todas las dudas que } \\
\text { tenía }\end{array}$ & 0.80 \\
\hline & & & $\begin{array}{l}\text { La persona que me atendió terminó el } \\
\text { trabajo rápidamente }\end{array}$ & 0.46 \\
\hline & & & $\begin{array}{l}\text { Me prestaron ayuda desde el principio } \\
\text { hasta el fin }\end{array}$ & 0.70 \\
\hline & & & Me solucionaron el problema & 0.83 \\
\hline & & & $\begin{array}{l}\text { Me describieron el problema y la } \\
\text { solución }\end{array}$ & 0.67 \\
\hline \multicolumn{5}{|c|}{$\%$ de explicación de la varianza total: $34.43 \%$} \\
\hline \multirow{4}{*}{2} & \multirow{4}{*}{$\begin{array}{l}\text { Comportamiento del } \\
\text { proveedor }\end{array}$} & \multirow{4}{*}{$\begin{array}{l}\text { Grado hasta el cual } \\
\text { el proveedor se } \\
\text { comporta de } \\
\text { manera afable y } \\
\text { dedicada mientras } \\
\text { trabaja con el } \\
\text { usuario }\end{array}$} & $\begin{array}{l}\text { Dejaron el servicio empezado y } \\
\text { volvieron al rato }\end{array}$ & 0.69 \\
\hline & & & $\begin{array}{l}\text { La persona que me atendió no fue } \\
\text { amable }\end{array}$ & 0.86 \\
\hline & & & $\begin{array}{l}\text { La persona que me atendió perdió } \\
\text { tiempo en actividades que no tenían } \\
\text { que ver con el servicio } \\
\end{array}$ & 0.56 \\
\hline & & & $\begin{array}{l}\text { La persona que me atendió prestó } \\
\text { atención cuando le hablé }\end{array}$ & 0.79 \\
\hline \multicolumn{5}{|c|}{$\%$ de explicación de la varianza total: $13.17 \%$} \\
\hline \multirow[b]{2}{*}{3} & \multirow[b]{2}{*}{ Tiempo de reacción } & \multirow{2}{*}{$\begin{array}{l}\text { Grado hasta el cual } \\
\text { el proveedor } \\
\text { reacciona a tiempo } \\
\text { ante la solicitud del } \\
\text { usuario }\end{array}$} & $\begin{array}{l}\text { Cuando solicité el servicio me } \\
\text { atendieron antes de } 24 \text { horas }\end{array}$ & 0.88 \\
\hline & & & $\begin{array}{l}\text { Tuve que esperar mucho tiempo para } \\
\text { que vinieran a atenderme }\end{array}$ & 0.82 \\
\hline \multicolumn{5}{|c|}{$\%$ de explicación de la varianza total: $9.94 \%$} \\
\hline \multirow[b]{2}{*}{4} & \multirow[b]{2}{*}{ Disponibilidad } & \multirow{2}{*}{$\begin{array}{l}\text { Grado hasta el cual } \\
\text { el usuario puede } \\
\text { ponerse en } \\
\text { contacto con el } \\
\text { proveedor }\end{array}$} & $\begin{array}{l}\text { La página Web estaba caída cuando } \\
\text { necesité el servicio }\end{array}$ & 0.87 \\
\hline & & & $\begin{array}{l}\text { Pude contactarme fácilmente con el } \\
\text { DRAI para solicitar el servicio }\end{array}$ & 0.79 \\
\hline \multicolumn{5}{|c|}{$\%$ de explicación de la varianza total: $7.1 \%$} \\
\hline 5 & $\begin{array}{l}\text { Oportunidad del } \\
\text { apoyo }\end{array}$ & $\begin{array}{c}\text { Grado hasta el cual } \\
\text { el trabajo se presta } \\
\text { en una hora } \\
\text { conveniente para el } \\
\text { usuario }\end{array}$ & $\begin{array}{l}\text { Me prestaron el servicio a una hora } \\
\text { conveniente para mi }\end{array}$ & 0.69 \\
\hline \multicolumn{5}{|c|}{$\%$ de explicación de la varianza total: $6.55 \%$} \\
\hline \multicolumn{5}{|c|}{$\%$ de explicación de la varianza total de las cinco dimensiones: $71,19 \%$} \\
\hline
\end{tabular}


Figura 2. Puntuación de los elementos ítems la dimensión capacidad de apoyo

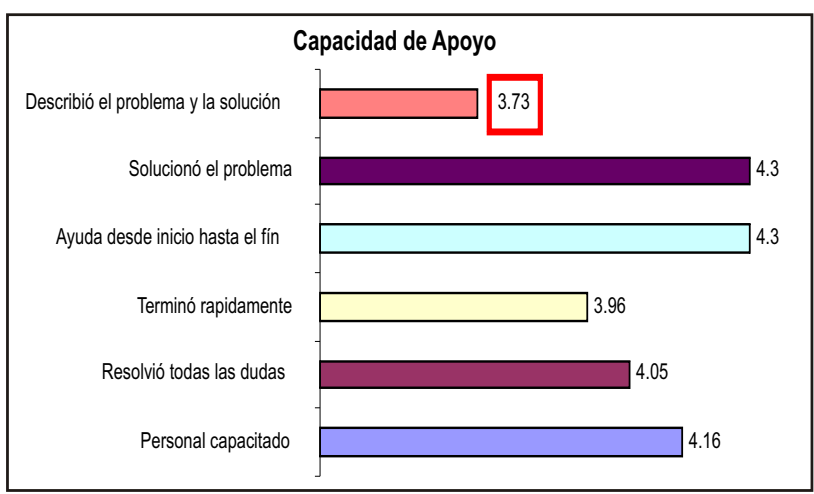

todos los atributos del servicio fueron redactados en forma de aseveraciones favorables. En este sentido, el mencionado puntaje puede tomar valores entre $1 \mathrm{y}$ 5 , donde 1 significa que los usuarios están totalmente en desacuerdo con el enunciado del ítem y 5 totalmente de acuerdo; de la misma manera, 1 deduce una actitud totalmente desfavorable hacia el atributo y 5 totalmente favorable.

En la figura 2 se muestra la puntuación de los ítems que conforman la dimensión capacidad de apoyo, la cual se refiere al grado hasta el que el proveedor demuestra capacidad para terminar el trabajo total.

De la figura 2 se deduce que, el servicio de soporte técnico ofrecido por el DRAl, tiende a cumplir satisfactoriamente con cada uno de los aspectos de la dimensión capacidad de apoyo, que son importantes para los usuarios, notándose que los atributos del servicio mejor evaluados son: la ayuda desde el principio hasta el fin por parte del proveedor y la solución del problema, ambos con puntaje de 4,3. De dicho cuadro se observa además, que los usuarios no están tan de acuerdo en que el proveedor les describe el problema y la solución, como lo están con los

Figura 4. Puntuación de los elementos de satisfacción de la dimensión de tiempo de reacción

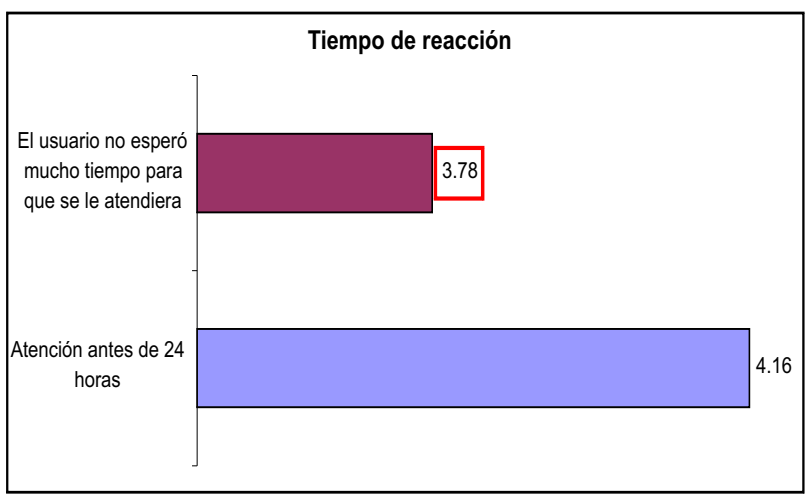

Figura 3. Puntuación de los elementos de satisfacción de la dimensión comportamiento del proveedor

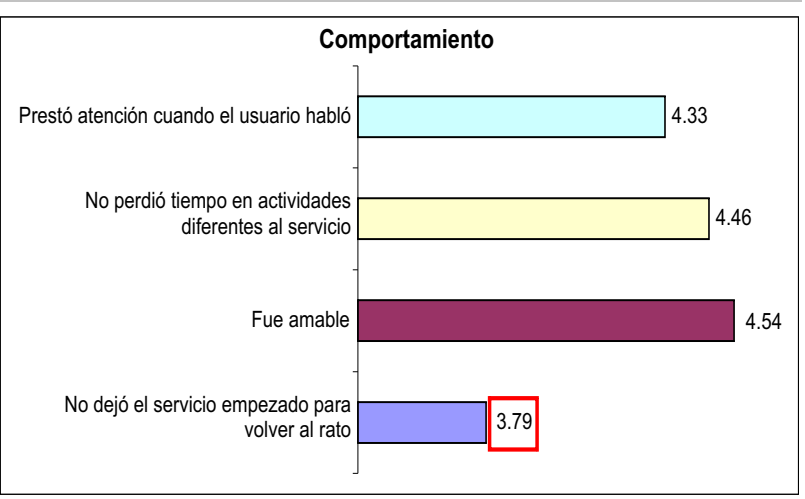

demás atributos del servicio, pues si bien, este requerimiento tiende a cumplirse satisfactoriamente, presenta la menor valoración $(3,73)$.

En la figura 3 se muestra la puntuación de las aseveraciones que conforman la dimensión comportamiento, que corresponde al grado hasta el cual el proveedor muestra un comportamiento afable y dedicado mientras trabaja con el usuario. Aquí se observa que los usuarios consideran que el DRAI, cumple satisfactoriamente con los aspectos que hacen alusión a la dimensión comportamiento del proveedor, siendo el trato amable el atributo mejor percibido $(4,54)$ y, la continuidad en la prestación del servicio, el atributo que consideran, se cumple en menor grado $(3,79)$.

En la figura 4 se muestra la valoración de los dos aspectos que conforman la dimensión tiempo de reacción, la cual se refiere al grado hasta el cual el proveedor reacciona a tiempo ante la solicitud del usuario.

De la figura 4 se deduce que, los usuarios muestran estar totalmente de acuerdo en que una vez solicitan

Figura 5. Puntuación de los elementos de satisfacción de la dimensión disponibilidad

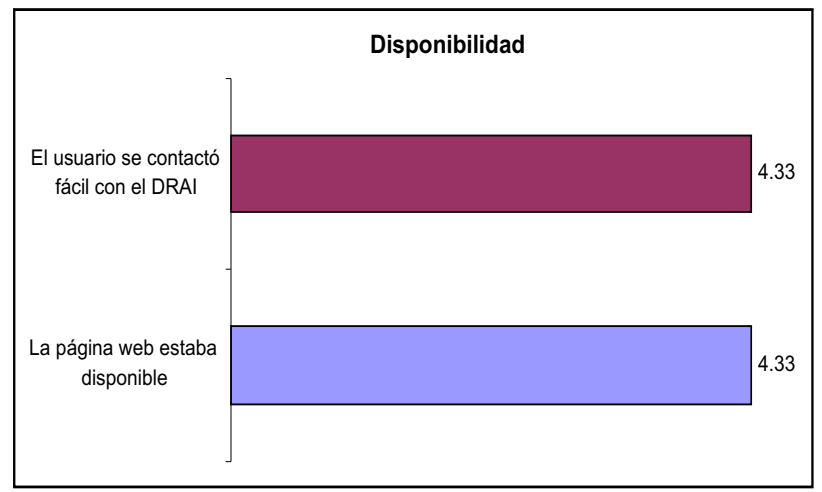


el servicio de soporte técnico, se les atiende antes de las 24 horas $(4,16)$; no obstante, la atención antes de dicho tiempo no parece considerarse rápida, comparada con la idea que tienen de lo que es esperar poco tiempo $(3,78)$.

La figura 5 ofrece la puntuación de los atributos de la dimensión disponibilidad del servicio, la cual hace referencia al grado hasta el cual el usuario puede ponerse en contacto con el proveedor.

De la figura 5 puede observarse que los usuarios perciben, que efectivamente el servicio de soporte técnico del DRAl, se caracteriza por permitir al usuario contactarse fácilmente cada vez que requiere apoyo y, dada la naturaleza del servicio, este contacto se efectúa principalmente a través de la Web. Ambos atributos con una puntuación de 4,33.

Con relación a la oportunidad del servicio, dimensión conformada por un solo ítem ("me atendieron a una hora conveniente para mi"), se encontró que los usuarios se sienten satisfechos con la hora en la que se les ha prestado el soporte técnico, pues este ítem fue valorado en 4,1 .

Finalmente, en figura 6 se muestra el grado de satisfacción global de los usuarios con las dimensiones de calidad.

De la figura 6 puede verse que en general, todas las dimensiones de calidad fueron evaluadas de manera favorable por los usuarios, obteniéndose puntuaciones que van desde 3.9 hasta 4.3 , siendo las mejores evaluadas las dimensiones disponibilidad del apoyo y comportamiento del proveedor, y la de menor puntaje, el tiempo de reacción.

Figura 6. Satisfacción de los usuarios con las dimensiones de calidad

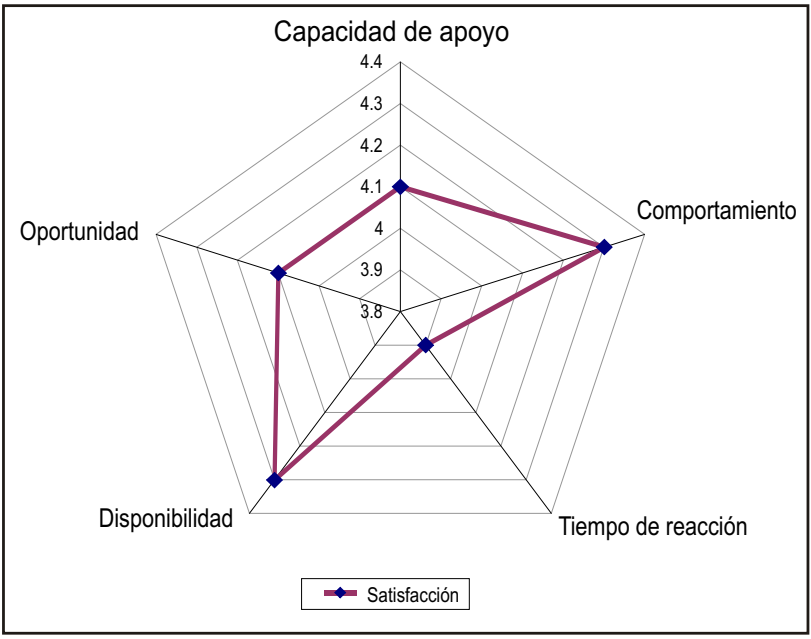

\section{Importancia relativa de las dimensiones de calidad}

En la figura 7 se presenta el diagrama de barras de la importancia relativa de las dimensiones de calidad del servicio.

En dicha figura se observa que la dimensión capacidad del apoyo es la más importante para los usuarios, puesto que dado el cálculo para tal fin (ver núm. 3.9), es la más relacionada con el juicio global del usuario acerca de la calidad percibida.

\section{Nivel de calidad del servicio}

De acuerdo con la ecuación 4, el nivel de calidad del servicio de soporte técnico esta dado por el siguiente modelo:

$$
C=0.39 V 1+0.24 V 2+0.15 V 3+0.07 V 4+0.15 V 5
$$

Ante las condiciones de operación que caracterizaban al servicio al momento de realizar la presente investigación, el grado de satisfacción de los usuarios es de 4,1, el cual, dada la definición de calidad adoptada en este estudio ("grado en que se satisfacen las necesidades de los usuarios"), corresponde también al nivel de calidad del servicio de soporte técnico del DRAI.

Relacionando la escala empleada en este estudio con los estados de satisfacción que plantea la literatura para el constructo subyacente "satisfacción" (ver cuadro 1), de la figura 9 se observa que la calidad del servicio de soporte técnico satisface las necesidades de los usuarios (4.1).

Figura 7. Importancia relativa de las dimensiones de calidad del servicio de soporte técnico

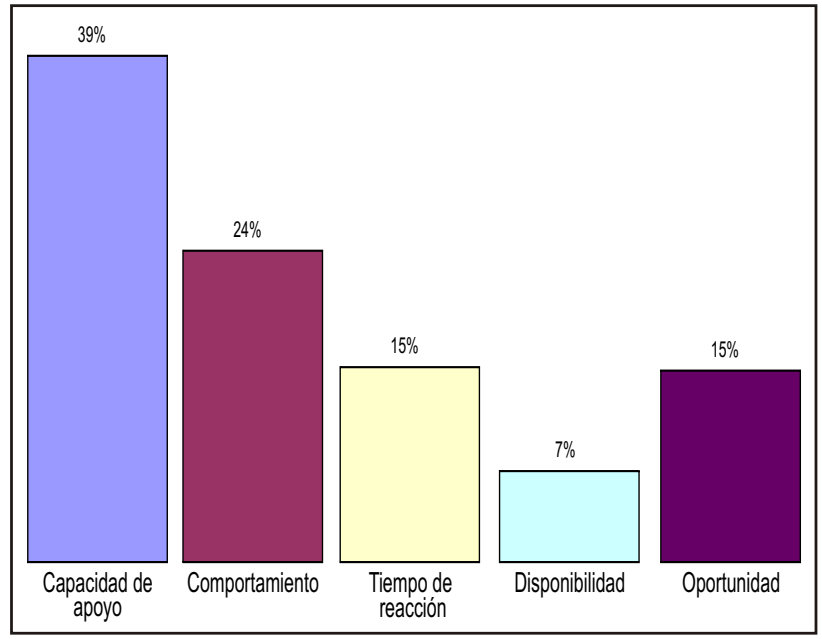


Figura 8. Nivel de calidad del servicio de soporte técnico del DRAI

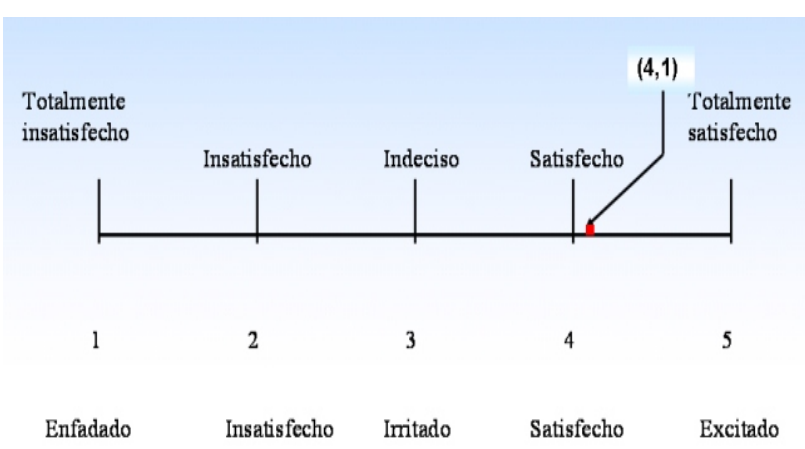

De acuerdo con los resultados presentados en este artículo, la calidad del servicio de soporte técnico del DRAI es satisfactoria para los usuarios $(4,1)$; sin embargo, en busca de la excelencia sostenida del servicio, siempre será fundamental generar esfuerzos para aumentar el valor de dicho indicador. Vale resaltar que, la descripción del problema y la solución $(3,73)$, la no interrupción de la prestación del servicio $(3,79)$ y, el poco tiempo que tiene que esperar el usuario para ser atendido $(3,78)$, son los atributos cuyo cumplimiento presentaron las percepciones menos favorables por parte de los usuarios, todas ellas valoradas por debajo de 4,0.

Una decisión que lamentablemente se toma en la mayoría de las organizaciones, es dirigir las acciones de mejoramiento hacia los atributos de menor puntuación; no obstante, esta decisión es apresurada porque una puntuación desfavorable no implica que dichos atributos sean los más importantes para los clientes, y de ser éste el caso, se generarán pérdidas importantes para la organización, puesto que los recursos son limitados, se emplea tiempo del personal y se invierte dinero, no logrando a pesar de ello, un aumento considerable en la calidad del servicio.

Ahora bien, siendo coherentes con la literatura (Hayes, B. 1999), las acciones de mejoramiento del servicio deberían orientarse hacia atributos de la dimensión capacidad de apoyo, puesto que es la más importante para los usuarios (ver figura 7). Sin embargo, a pesar que el procedimiento visto en este artículo permite conocer lo que desean los usuarios, como lo desean, la importancia que le asignan a ello, y de igual manera, permite contar con indicadores de eficacia del servicio; el análisis de la calidad, si bien es una exigencia para las organizaciones, debe verse como un primer paso hacia el análisis de la confiabilidad, pues ésta última constituye una práctica aún más efectiva para las organizaciones, por considerar las fallas del servicio, la severidad de las mismas y el comportamiento que caracteriza a la calidad a lo largo del tiempo, y en consecuencia, permite identificar con mayor certeza hacia donde deben orientarse los esfuerzos del talento humano y los recursos de la organización (Pérez, Rave, J. et al 2006).

Lo anterior debido a que, en el caso del servicio de soporte técnico, siendo la capacidad de apoyo la dimensión más importante para los usuarios, no necesariamente implica que ella, a lo largo del tiempo, sea la que más fallas presenta, entendiéndose falla como el evento de no satisfacer al usuario.

Esto implica no sólo analizar los indicadores contemplados en análisis típicos sobre calidad de servicios o sobre satisfacción del cliente, puesto que los promedios empleados para tal fin pueden ser favorables, y al mismo tiempo, esconder situaciones no deseadas para la organización, que si bien, de manera general parecen no existir, realmente hacen presencia y sus efectos negativos tarde o temprano ameritarán intervenciones.

Ejemplo de lo dicho es un cliente insatisfecho, que a pesar de ser una minoría, cuya percepción sobre el servicio no afecta considerablemente el promedio de satisfacción de un amplio conglomerado de clientes, sí constituye una fuente potencial de publicidad boca a boca sobre aspectos desfavorables de la organización, sobrando resaltar las consecuencias que ello implica para su supervivencia.

\section{CONCLUSIONES}

Lo visto en este artículo, constituye los primeros resultados de aplicar, al servicio de soporte técnico del DRAI, una metodología desarrollada por los autores para evaluar y analizar la confiabilidad de servicios desde la perspectiva del cliente (Pérez, Rave, J. et al 2006). Dicha metodología integra elementos de: estadística multivariable, investigación cualitativa, calidad de servicios, teoría de la probabilidad y confiabilidad de sistemas.

La aplicación de la mencionada metodología al servicio de soporte técnico, permitió proponer directrices de mejoramiento fundamentadas en el análisis de las causas de las fallas que mayor incidencia negativa ejercen sobre la calidad y la confiabilidad del servicio, a fin de eliminarlas $y$ asegurar que sus efectos no vuelvan a repetirse. Estas directrices fueron establecidas considerando tanto la voz del usuario como la del proveedor. 
Igualmente, el uso de la metodología le posibilitó al Departamento de Recursos de Apoyo e informática (DRAI), identificar diversas situaciones no deseadas, reflexionar sobre la posición que ocupa en cuanto a calidad y confiabilidad se refiere, definir objetivos retadores, e implementar estrategias de mejoramiento orientadas a garantizar en el tiempo, la plena satisfacción de sus usuarios.

Al mismo tiempo, la metodología desarrollada se considera lo suficientemente robusta para ser aplicada a otros tipos de servicios, y constituye un aporte al vacío de conocimiento existente en el área de la confiabilidad en los servicios.

\section{REFERENCIAS BIBLIOGRÁFICAS}

1. Andriani, C., Biasca R. y Rodríguez M. (2003). Un nuevo sistema de gestión para lograr Pymes de clase mundial. Ed. Norma, México. P. 112.

2. Bolton, R. y Drew, J. (1991). A multistage model of customer' assessments of service quality and value». Journal of Consumer Research. Vol. 17, Marzo. P. 375-384.

3. Carmines, E. y Zeller, R. (1979). Reliability and validity assessment. Beverly Hills, Cal.: Sage Publications, Serie "Quantitative Applications in the Social Sciences". Vol. 17.

4. Chang, L. (1994). A psychometric evaluation of 4point and 6-point Likert-type scales in relation to reliability and validity. Applied psychological measurement. Vol.18 (3). P. 205-215.

5. Chias, J. (1999). El mercado todavía son personas. Editorial McGraw Hill. España. P.19.

6. Churchill, G. y Surprenant, C. (1982). An investigation into the determinants of customer satisfaction. Journal of Marketing Research. Vol.19, Noviembre. P. 491-504.

7. Dallas, E. (1998). Métodos multivariados aplicados al análisis de datos. Ed. Thomson, México.
8. Escobar R. et al (2003). Confiabilidad: historia, estado del arte y desafíos Futuros. Revista DYNA, Nro. 140, Medellín, Noviembre. P. 5-21.

9. Hayes, B. (1999). Como medir la satisfacción del cliente: desarrollo y utilización de cuestionarios. 2.ed. España.

10. Juran, J. y F. Gryna (1995). Análisis y Planeación de la Calidad. Mc Graw Hill, México.

11. Kennedy, D. y Young B. (1989). Managing quality in staff areas. Quality Progress. Vol.22 (10).

12. Larrea, P. (1991). Calidad de Servicio. Del marketing a la estrategia. Díaz de Santos, Madrid. P. 75.

13. Likert, R. (1932). A Technique for the measurement of attitudes. Archives of Psychology, Vol. 40. P. 1-55.

14. Montgomery, D.(1996). Introduction to Statistical Quality Control, John Wiley and Sons, USA

15. Oskamp, S. (1977). Attitudes and opinions. Englewood Cliffs, N. J. Prentice Hall.

16. Padua, J. (1979). Técnicas de investigación aplicadas a las ciencias socials. México: El Colegio de México/Fondo de Cultura Económica.

17. Parra, C. et al (2000). Análisis exploratorio de factores incidentes en la evaluación docente por parte de los estudiantes. Revista Lectiva. Universidad de Antioquia. Vol.1 (4). P. 63-75.

18. Pérez, C. J. (2002). Medida de la satisfacción de los clientes, dificultades y errores.

19. Pérez, Rave. J. et al (2006). Confiabilidad del servicio de soporte técnico del DRAl desde la perspectiva del usuario. Departamento de Ingeniería Industrial. Universidad de Antioquia.

20. Thurstone, L. (1931). The measurement of social attitudes. Journal of Abnormal and Social Psychology. Vol.26. P. 249-269. 